\title{
A practical guide to filtering and prioritizing genetic
} variants

\author{
Mahjoubeh Jalali Sefid Dashti and Junaid Gamieldien \\ South African National Bioinformatics Institute, University of the Western Cape, Bellville, South Africa
}

BioTechniques 62:18-30 (January 2017) doi 10.2144/000114492

Keywords: whole-genome sequencing; whole-exome sequencing; functional variants; variant prioritization

Next-generation sequencing (NGS) of whole genomes and exomes is a powerful tool in biomedical research and clinical diagnostics. However, the vast amount of data produced by NGS introduces new challenges and opportunities, many of which require novel computational and theoretical approaches when it comes to identifying the causal variant(s) for a disease of interest. While workflows and associated software to process raw data and produce high-confidence variant calls have significantly improved, filtering tens of thousands of candidates to identify a subset relevant to a specific study is still a complex exercise best left to bioinformaticists. However, as this prioritization procedure requires biological/biomedical reasoning, biologists and clinicians are increasingly motivated to handle the task themselves. Here, we describe a set of guidelines, tools, and online resources that can be used to identify functional variants from whole-genome and whole-exome variant calls and then prioritize these variants with potential associations to phenotypes of interest. Insights gained from a recently published analysis of protein-coding gene variation in $>60,000$ humans by the Exome Aggregation Consortium (ExAC) are also taken into account.

Next-generation sequencing (NGS) technologies and applications, such as whole-genome sequencing (WGS) and whole-exome sequencing (WES), are increasingly used in the study of Mendelian, rare complex, and genetically. heterogeneous disorders. Early successes in making actionable genetic diagnoses of Mendelian disorders showed that WES has exceptional potential for empowering biomedical research and clinical diagnostics (1-5). Uptake has been extremely rapid, with PubMed reporting (at the time this review was written) 1646 papers containing the term "exome sequencing" in the title and 6077 papers mentioning the term anywhere in the text.

While sequencing technology platforms, reference data sets, software, and analysis pipelines have significantly improved since the early days of NGS, standards and guidelines for prioritizing genetic variant candidates likely to be relevant in disease research are still in their infancy. WESand WGS-based experiments generate data on large numbers of genetic variants, many of which, in the context of a disease study, are not relevant since they are very unlikely to have a functional effect at the protein and/or systemic level. Nonetheless, there is no single "recipe" that can be used for identifying functional variants. Every filtering strategy inevitably starts with multiple seemingly logical assumptions regarding the properties of causal variants, which when combined with overreliance on automation and predictive tools may either discard relevant candidates too early or produce excessive false positives. It is also important to note that any given individual's exome contains a large number of protein sequence-altering inherited genetic variations and de novo mutations, but only a small fraction of these can be expected to impact protein function (e.g., nonsense mutations that result in a truncated protein). A smaller number of these mutations are likely to be deleterious or relevant to a disease study, even if they significantly alter protein function or expression levels, due to incomplete or non-penetrance.

Computational algorithms are currently used to predict the potential functional impact and relevance of variants, but due to the sheer volume of data, extreme caution should be exercised when weighing the importance of these predic- tions. A study by Miosge et al. (2015) demonstrated that even for de novo or rare missense mutations found by genome sequencing, nearly half inferred as deleterious were in fact nearly neutral mutations, based on data from functional genomics studies (6). Furthermore, the software and the reference transcript set used for annotating variants as exonic, at splice sites, missense, etc., have been shown to have a large effect on the ultimate variant annotations from a WES/WGS study, which then has bearing on downstream filtering and functional impact assessments.

Recent studies have contributed greatly to the establishment of guidelines for investigating the potential causality of sequence variants in human disease (7), as well as the specialized requirements for identification of causal variants and disease diagnosis in a clinical setting (8). The ExAC consortium recently published data and findings based on the sequencing of nearly 65,000 human exomes that can be valuable in the development of rules and protocols for the efficient filtering of candidate diseasecausing variants (9). Here, we describe a series of guidelines for developing appro- 


\section{Functional Variant Identification}

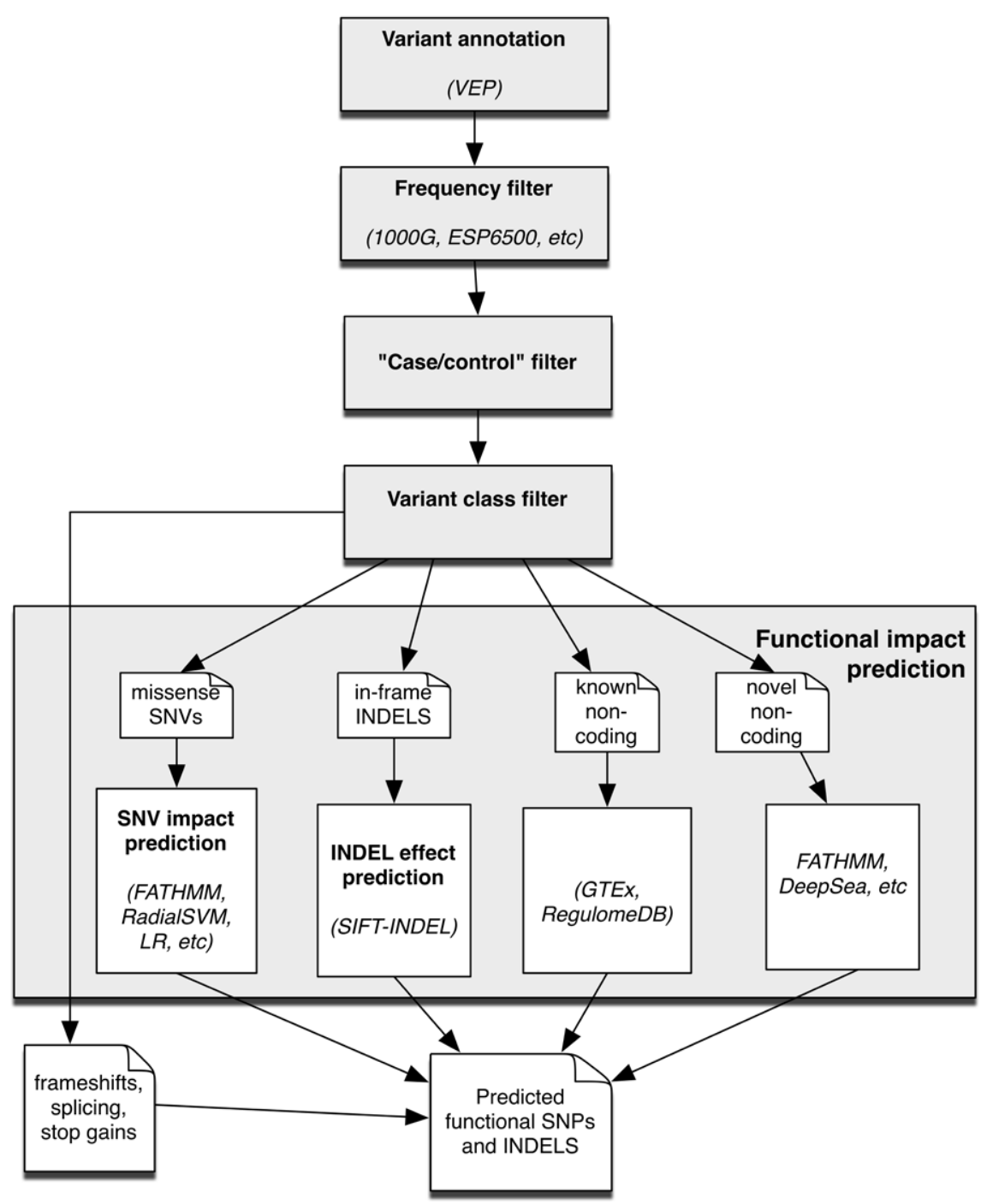

Figure 1. Identifying likely function-impacting variants. Variants are filtered according to category, and those that cannot be immediately assigned as loss-of-function are further characterized using algorithms for predicting functional impact.

priate variant prioritization strategies that can be applied to a genome- or exomescale data set to identify candidates for further study. Our aim is not to define absolute rules but rather to provide guidelines that can be adjusted or replaced as genomic knowledge and software improve. It is important to note that these recommendations are for research use only and, therefore, do not necessarily conform to the above-mentioned interpretation guidelines suggested by clinical sequencing regulatory and advisory bodies. Finally, it is important to note that false-positive variant calls are still a reality, and therefore any candidates should be independently verified using the current gold standard, Sanger sequencing, before proceeding with further analyses.

\section{Identifying candidate}

\section{function-impacting variants}

A. Variant annotation

Variant annotation involves adding auxiliary metadata and knowledge to quality-filtered raw putative variant calls in an effort to enhance assessment of variants likely to impact function. While information regarding known allele frequencies, involvement in human diseases and phenotypes, and predicted deleterious effects are commonly added, the most fundamental annotations will also categorize variants based on their relationships to genes, their transcripts, and other central sites (Figure 1). features such as exons, introns, and splice

\section{BIOSPAA SYSTEM FOR AUTOMATED LIVE CELL ANALYSIS}

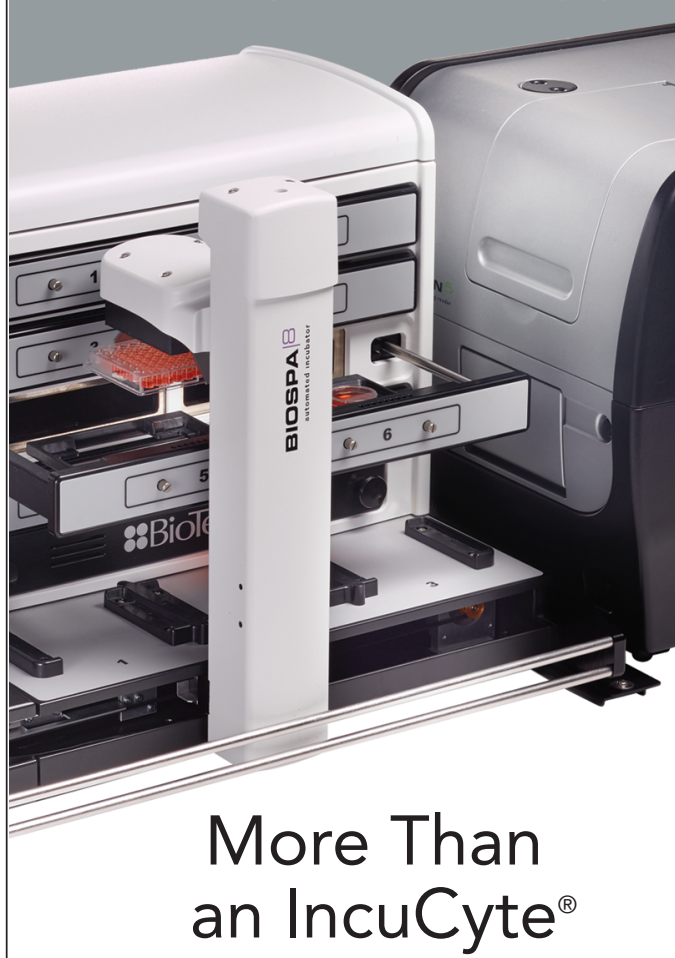

\begin{tabular}{|c|c|c|}
\hline & $\begin{array}{l}\text { BioSpa }^{\mathrm{TM}} \\
\text { System }\end{array}$ & $\begin{array}{c}\text { IncuCyte }{ }^{\circledR} \\
\text { Zoom }\end{array}$ \\
\hline \multicolumn{3}{|l|}{$\begin{array}{l}\text { Live cell } \\
\text { imaging }\end{array}$} \\
\hline $\begin{array}{r}\text { Temperature, } \\
\text { humidity, } \\
\text { gas control }\end{array}$ & $\begin{array}{l}\text { Included, } \\
\text { monitored }\end{array}$ & $\begin{array}{l}\text { Incubator } \\
\text { required }\end{array}$ \\
\hline $\begin{array}{l}\text { Labware } \\
\text { capacity }\end{array}$ & 8 & 6 \\
\hline $\begin{array}{r}\text { Objective } \\
\text { capacity }\end{array}$ & 6 & 1 \\
\hline $\begin{array}{r}\text { Magnification } \\
\text { range }\end{array}$ & $\begin{array}{c}1.25 x \text { to } \\
60 x\end{array}$ & $4 x$ to $20 x$ \\
\hline $\begin{array}{r}\text { Fluorescence } \\
\text { colors } \\
\text { available }\end{array}$ & $>15$ & 2 \\
\hline \multicolumn{3}{|l|}{$\begin{array}{r}\text { Z-stacking } \\
\text { for 3D } \\
\text { biology }\end{array}$} \\
\hline \multicolumn{3}{|l|}{$\begin{array}{r}\text { Laser } \\
\text { autofocus }\end{array}$} \\
\hline \multicolumn{3}{|l|}{$\begin{array}{r}\text { Advanced } \\
\text { cell-level } \\
\text { analysis }\end{array}$} \\
\hline \multicolumn{3}{|l|}{$\begin{array}{r}\text { Washing, } \\
\text { media } \\
\text { replacement }\end{array}$} \\
\hline \multicolumn{3}{|l|}{$\begin{array}{r}\text { Reagent } \\
\text { dispensing }\end{array}$} \\
\hline \multicolumn{3}{|c|}{ www.biotek.com/biospasystem } \\
\hline \multicolumn{3}{|c|}{ Think Possible } \\
\hline
\end{tabular}


(i) Choice of reference transcripts. Since the functional consequences of any variant (e.g., intronic rather than nonsynonymous exonic) can differ significantly depending on which transcript isoform is analyzed, the choice of reference is very important. The significance of this choice was shown convincingly by McCarthy et al. (10), who quantified differences in the annotation of $>80$ million variants from a WGS study using the RefSeq (11) and Ensembl (12) transcript data sets. Since that analysis found that a large number of potential loss-of-function (LoF) variants were not detected using RefSeq, we recommend using Ensembl transcripts for variant annotation, regardless of the software tool used.

(ii) Variant annotation tools. Several computational variant annotation tools that produce integrated reports that can be used for further rule-based filtering have been developed. SnpEff (13), ANNOVAR (14), and the Variant Effect Predictor (VEP) (15) are arguably the most widely used, with the latter gaining prominence. All three applications enable variant annotation based on Ensembl transcripts and also leverage the rich annotations in dbNSFP (16), a database of curated annotations and functional effect predictions for all potential nonsynonymous and splice-site single-nucleotide variants in the human reference genome. However, due to the significant advantages over ANNOVAR identified by McCarthy et al. (10), particularly when annotating frameshift, nonsense, and splicing variants, we base our guidelines here on VEP and recommend its use.

VEP is free for both non-commercial and commercial use and is available as a standalone version under the Apache 2.0 license via an intuitive web server (www.ensembl.org/Tools/VEP) or through an API. VEP has a flexible plugin architecture enabling algorithmic extensions for additional analysis. For example, an experimental plugin has been developed to indicate expression levels across tissues for many transcripts, which can be used to prioritize variants based, for example, on a tissue targeted by the disease of interest. VEP produces reports in several standard formats as well as a customizable output and annotates SNVs and indels with allele frequencies in public databases, gene/transcript effects, and site conservation scores and predicted functional impact scores and classifications based on dbNSFP. Downstream filtering is also simplified through assignments of annotations such as zygosity, gene symbols, the genomic location of the variants [i.e., exonic, intronic, intergenic, UTR, splice site (donor, acceptor), and transcription factor binding site], as well as their classes, including synonymous, missense, and frameshift variants. All versions are compatible with the VCF format produced by majority of the variant calling tools, including the widely used Genome Analysis Toolkit (17) developed by the Broad Institute. As one output format is tab-delimited text, most of the downstream filters described below can be easily implemented in a spreadsheet software package such as Microsoft Excel.

B. Inheritance and statistical filtering MacArthur et al. (18) reinforced the important point that strong genetic support is paramount for assigning likely causality to new gene variants identified using WES, WGS, or similar approaches. Only then should ancillary support from functional and bioinformatics studies be added to support the candidate. Working with an inheritance model or expected segregating variant frequency threshold is a useful way to filter out and reduce the number of variants that have to be analyzed downstream. In both filtering scenarios, delayed onset, incomplete penetrance, and/or variable expressivity of the clinical phenotype should be taken into account when no obvious candidates remain after filtering, and rules and thresholds should be adjusted before terminating the discovery exercise as unsuccessful, or in the case of WES, prematurely concluding that a causal variant is in the non-coding portion of the genome.

In an autosomal dominant inheritance model, candidates are heterozygous variants seen in all affected individuals in a family, while unaffected individuals are homozygous for the reference allele. In diseases with autosomal recessive inheritance, the pattern is, most frequently, heterozygous for two different pathogenic mutations in all of the affected individuals in a family when parents are non-consanguineous or, less frequently, homozygous. Unaffected parents are heterozygous for one allele, while other unaffected family members are either heterozygous or homozygous for the reference allele.

Other scenarios include disorders that occur as a result of spontaneous mutation, where the allele in the affected individual is not present in either parent (private mutation), as well as the somatic mutations seen in most types of cancer, where the variants are seen in the tumor genome/exome but not in a matching normal sample from the same individual. The ability to perform this filtering step is, however, dependent on the availability of genomes/exomes from unaffected family members or from matching normal tissue from the same individual. An important consideration is whether the working family pedigree is accurate. It should be noted that when studying polygenic disorders or population genetic studies, these criteria cannot be applied. Rather, variants should be assessed for statistically different frequencies in cases versus controls or between population groups using techniques such as exome-wide association (19).

VAAST (20) (www.yandell-lab.org/ software/vaast.html), GEMINI (21) (http:// gemini.readthedocs.io/en/latest/), and KGGSeq (22) (http://jjwanglab.org/ wkggseq) are flexible software frameworks that perform variant annotation as well as filtering based on a user-provided pedigree file and specified inheritance model. Filtering is more sophisticated than ANNOVAR's simple check for one likely pathogenic variant in an autosomal dominant model and two in a recessive model without using a pedigree. The first two tools enable prioritization using several of the variantbased and gene-based criteria that will be described below, allowing users to explore existing hypotheses about inheritance and underlying biology in a reasonably intuitive manner. It should be noted that these automated tools all have limitations and that it is often advantageous to filter a VEP annotated variant list in a stepwise manner using an appropriate combination of the concepts detailed in this article.

\section{Variant class filtering}

Once a set of variants is selected based on correct segregation with the target group or individual of interest, further selection can be carried out using annotations for the genomic location and the variant class, denoted in the "consequence" field of the VEP report. Exonic missense, nonsense, stop-loss, frameshift and splice site variants all have potential to affect protein function. While truncating and splicing mutations are of primary interest in disease-related studies due to their potentially high cellular 
and systemic impact, additional consideration is necessary when progressing these as candidates for further evaluation. The proportion of false positives from these classes can be expected to be significantly elevated due to a combination of uniformity in sequencing error and the rate of true loss-of-function variation being much lower due to selection constraints (7) While the presence of the same variant in multiple samples can provide some level of confidence, particularly if the data were generated from multiple sequencing runs, we recommend applying a combination of computational and manual assessment in the variant filtering process to avoid timeconsuming prioritization and hypothesis generation based on false positives. Here, we propose extracting reads spanning the genomic location of the variant, aligning them to the specific region in the reference genome using appropriate software, and then visually assessing whether reads have been correctly aligned and the variant call is likely to be real. Even then, should the candidate pass downstream prioritization, we recommend independent confirmation using Sanger sequencing before functional studies are done or any conclusion is made about the variant's possible importance.

It is very important to mention the phenomenon of tandem substitutions, which affect multiple nucleotides in a single codon and can have a profound impact on the transcript and/or protein but are missed by most variant callers, resulting in incorrect predictions of amino acid changes. The ExAC study (9) found on average 23 multinucleotide variants (MNV) per sample, including several instances where the effect of a protein-truncating variant (PTV) is eliminated by an adjacent SNP or apparently synonymous or missense variants result in a PTV. We recommend that variant call files be checked using the MAC (Multi-Nucleotide Variant Annotation Corrector) pipeline, which was specifically developed to rectify potentially misannotated MNVs (23).

True missense SNVs can additionally be subjected to functional impact prediction (discussed below).

\section{Population frequency filtering}

One of the primary criteria for predicting if a variant is likely to have a functional effect on the encoded protein is rarity (24). Here, a rare nonsense SNP can be expected to have a larger functional effect than a frequently occurring one. VEP provides allele frequencies for each variant, both as an average across all the populations studied and for individual population groups, including African, Admixed American, East Asian, Finnish, Non-Finnish European, South Asian, and other. The population frequency databases include the 1000 Genomes Project (5 ethnicity groups + average); approximately 6500 exomes from the NHLBI Exome Sequencing Project (http://esp.gs.washington.edu, 2 ethnicity groups + average); and the ExAC (http://exac.broadinstitute.org/), which includes exome sequencing data from several large-scale sequencing projects ( 7 ethnicity groups + average). Currently, we recommend that preference be given to the ExAC database due to its frequencies being calculated from an order of magnitude more samples than the second largest data set (9).

In disease studies, a commonly used starting point for filtering out variants is a minor allele frequency (MAF) >1\%. However, the majority of disease-causing variants are expected to display incomplete penetrance and/or variable expressivity of the clinical phenotype (25), which will invariably mean that there is a significant chance of carriers being labeled as healthy in case control data sets. Lazarin et al. (2013) reported that $24 \%$ of 5700 asymptomatic individuals

\section{Muscle_Skeletal eQTL rs491880 ENSG00000142657.16}

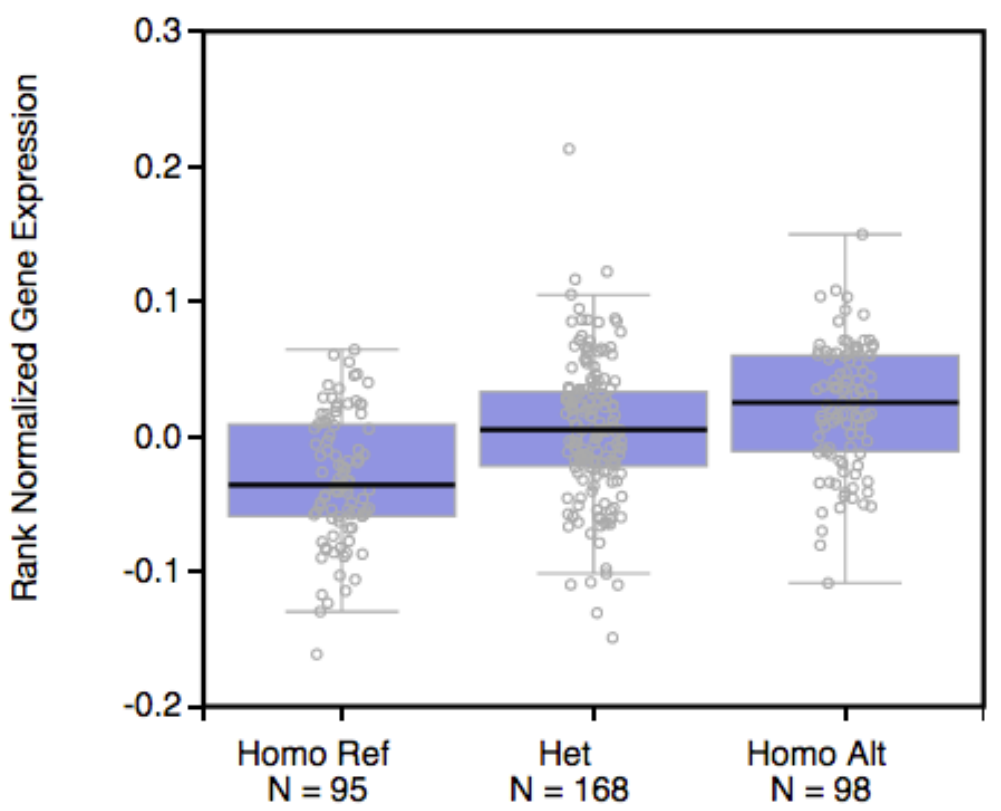

Figure 2. Effects of a common noncoding polymorphism on expression of the phosphogluconate dehydrogenase gene in human skeletal muscle. The graph illustrates the importance of considering common quantitative trait loci (eQTL) as potential disease modifiers, since both the homozygous (Homo Alt) and heterozygous (Het) forms result in higher basal expression compared to the reference allele (Homo Ref) and may thus impact cell biology. 


\section{Variant Prioritization}

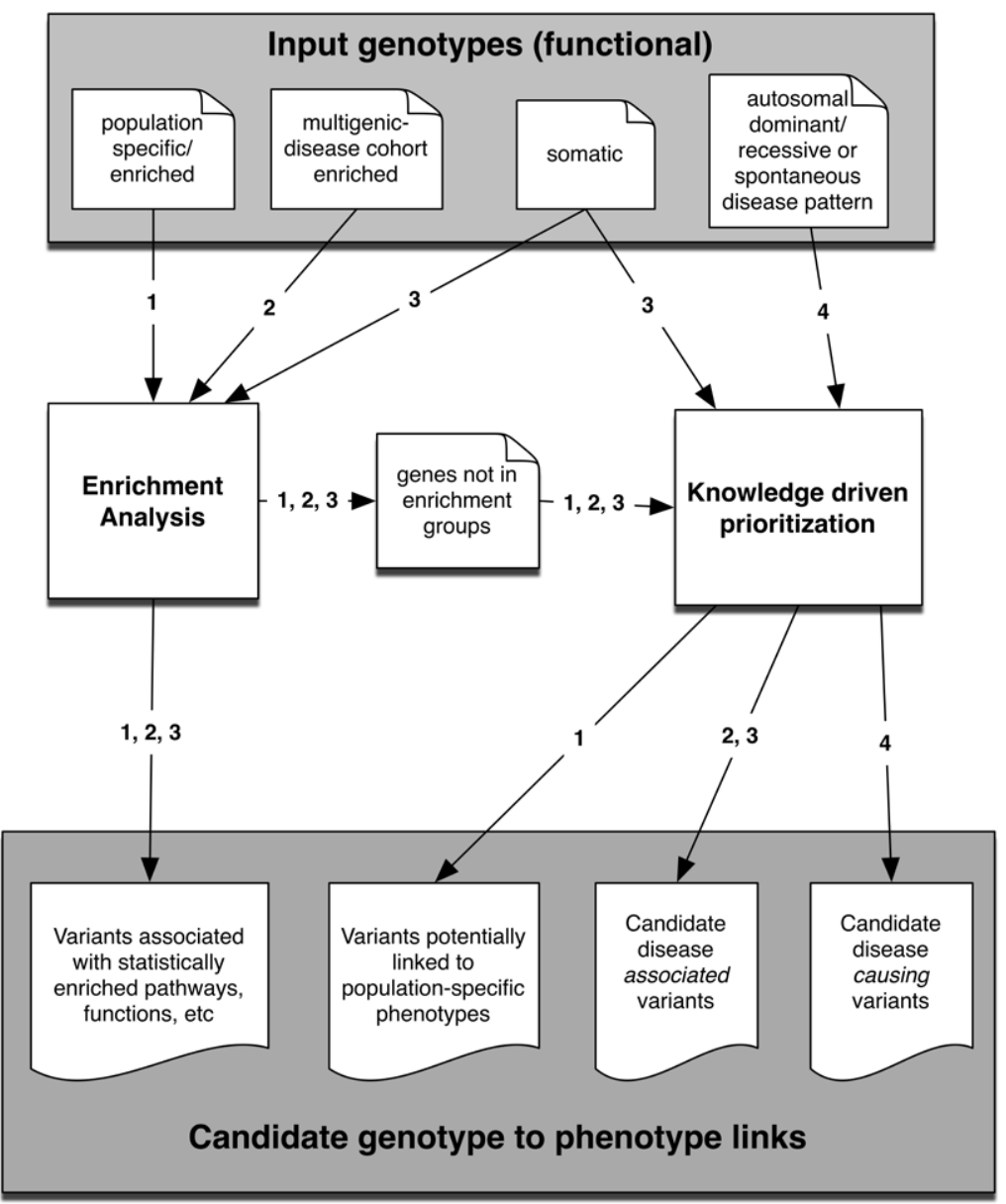

Figure 3. Prioritizing genes bearing functional variants. Extant knowledge about the gene and its functions is used to identify candidates that have likely relevance to the disease of interest.

pass the conservation threshold. It has been demonstrated that a significant fraction of known disease-causing alleles are fixed and neutral in the genomes of other species, modulated by a phenomenon referred to as compensated pathogenic deviation (27). Nevertheless, various permutations of these two annotation classes can be produced to identify variants that have the highest functional impact. For example, filters can be defined using the appropriate prediction shorthand from VEP ("T" or "B" for tolerated/benign and "D" or "P" for deleterious/pathogenic) and conservation score cut-offs (tool-specific). Depending on the research question, more stringent filters can be applied to identify variants with multiple "deleterious" annotations that are also found at a highly conserved site.

New variant effect predictors are constantly being developed; many are being added to dbNSFP and, thus, VEP. While the majority of publications still use a combination of SIFT (28) and PolyPhen (29), the specificities of these tools are extremely low $(13 \%$ and $\sim 16 \%$ respectively) $(30,31)$. Therefore, we recommend becoming familiar with the other tools and applying them in combination in the filtering pipeline to prevent discarding candidates due to false negatives. Tools such as Functional Analysis Through Hidden Markov Models (FATHMM), CADD, MutationTaster, MutAssessor, RadialSVM, likelihood ratios (LR), and the likelihood ratio test (LRT), as well as conservation prediction tools including Phylogenetic P-Values (PHYLOP), SiPhy, and Genomic Evolutionary Rate Profiling (GERP) scores (as suggested in published guidelines for each tool), can be used in combination for functional effect prediction.

There are no standard rules for these filters, which should be defined based on the hypothesized molecular mechanism of the disease. For example, in Mendelian disorders, it is reasonable to assume that the single causal variant will have a large effect size, so a very stringent filter is appropriate as a first pass. Relaxing the rules may, however, be appropriate when no candidates remain following strict filtering, since all functional prediction tools produce false negatives and, for example, novelty/ rarity together with the expected inheritance pattern and site conservation are already substantial evidence for implicating a variant as being potentially causal. If ranking of candidate missense SNVs is desired, multiplying all of the actual scores (also reported by VEP) and all of the conservation scores and sorting by that result will cause those cases where the majority of tools are in agreement to "bubble to the top." We prefer using RadialSVM and/or $L R$, as these ensemble methods combine scores and predictions in a much more sophisticated manner (31).

(ii) In-frame insertions and deletions. While rare in-frame indels may be deleterious, SIFT-indel (32) can be used to predict their functional impact (http://sift.bii.a-star. edu.sg/www/SIFT_indels2.html). Again, predicted deleterious effects should be treated with caution, and segregation with the appropriate diseased individuals or group and rarity in the general population should take precedence in prioritizing candidates from this variant class. Additionally, classical bioinformatics tools can be used to determine whether a candidate variant significantly disrupts or deletes an important protein domain. InterProScan (www.ebi. ac.uk/interpro/search/sequence-search), which utilizes the InterPro database (33), is an excellent tool for annotating the translated protein with detailed domain and functional signature information.

(iii) Known non-coding variants. Non-coding variants occur in introns, upstream or downstream of coding regions in $3^{\prime}$ and $5^{\prime}$ UTRs, and in intergenic regions. Many non-coding variants that have been assigned dbSNP identifiers are annotated by the Regulome database project based on whether they are located in and have a potential disruptive effect on regulatory elements and intergenic regions in the human genome, including regions of DNAase hypersensitivity, transcription factor binding sites, and biochemically characterized promoter regions. For example, this database was used to identify a functional intronic polymorphism that is predictive for post-surgery lung cancer survival (34). SNP identifiers can be submitted to the 
RegulomeDB (35) web server at www. regulomedb.org, which will assign them a category based on likely functional impact.

The Genotype-Tissue Expression (GTEx) project (36) is another resource that has cataloged a large number of tissue-specific and shared regulatory expression quantitative trait loci (eQTL) variants. GTEx has been used to identify signals from genomewide association studies (GWAS) that could be explained by eQTLs. This resource is invaluable for identifying genes whose expression is affected by genetic variation. This knowledge allows a researcher to make decisions on the gene's, and thus the variant's, potential involvement in the disease phenotype. eQTL signals in the database can be accessed via an intuitive web portal (www.gtexportal.org) or by downloading the data. One very useful GTEx feature is the illustration of differences in effect sizes between heterozygous and homozygous eQTL variants. In fact, simply exploring the database via the multiple interfaces can be instructive when it comes to thinking about variant effects including: (i) the down-regulation of expression of the target gene by functional variants and (ii) the likelihood that rare variants have a functional impact. Both these points are illustrated in Figure 2, which shows a GTEx single-tissue eQTL $\left(P=1.4 \mathrm{e}^{-13}\right)$ boxplot for rs491880, a commonly occurring variant in all HapMap populations (global MAF = 43.3\%; calculated from $>2000$ individuals).

(iv) Novel non-coding variants. WGS can also be used to survey non-coding variants, which make up the majority of variants in the genome. While most GWAS signals map to non-coding regions and potentially point to non-coding variants, the functional interpretation of this class of variants is challenging. Algorithmic methods to predict the functional effects of novel non-coding variants based on structural and regulatory properties are of importance, provided downstream validation is performed before making strong hypotheses. Prediction tools such as FATHMM-MKL (37) (http:// fathmm.biocompute.org.uk/), Combined Annotation-Dependent Depletion (38) (CADD) (http://cadd.gs.washington.edu/ upload/) or Genome Wide Annotation of Variants (GWAVA) (www.sanger.ac.uk/ sanger/StatGen_Gwava) can be used to assess likely functional impact. FATHMM$M K L$ has been shown to outperform CADD and GWAVA, and we recommend using it with a minimum score threshold of 0.9 to minimize false positives. We also recommend DeepSEA (http://deepsea. princeton.edu), which employs artificial neural network deep-learning of patterns that functionally affect chromatin (39) to improve prioritization of functional variants at single-nucleotide sensitivity, including eQTLs and disease-associated variants.

\section{Knowledge-driven variant prioritization}

Even with strong experimental design and variant filtering protocols, WGS and WES studies often produce many more candidates with likely functional effects than can be verified experimentally. While it is possible to rank variants based on predicted impact on a given protein, identification of the strongest candidate variants for a particular disease or phenotype is not always obvious. For this reason, assessing candidate genes possessing functional variants in the context of existing biomedical knowledge and their known biomolecular functions is an important step in producing a manageable set of variants for further validation or exploration. Depending on the study and the biological question at hand, candidates can be evaluated individually or as a set. Several factors should be considered at this point (Figure 3):

(i) Is the variant known or predicted to be involved in the disease of interest? VEP, recommended earlier for variant annotation and functional prioritization, produces annotations for ClinVar (www. ncbi.nlm.nih.gov/clinvar/), a public archive reporting associations between genomic variants and phenotypes. Using a set of identifiers and supporting evidence, ClinVar classifies variants having clinical significance as disease-causing (40).

(ii) Is the variant known to be involved in the disease or in a related disease? Mendelian Inheritance in Man (http:// omim.org) is an excellent resource for this information. Single genes can be searched using the HUGO gene symbol and selecting "Gene name" in the "Limits" menu. OMIM information for large gene sets can be programmatically obtained by accessing the API (see the "Tools" link at the aforementioned URL). DisGeNET (41) is another excellent resource that integrates expert-curated databases with text-mined data, covering information on Mendelian

\section{Table 1. Selected ontology annotations supporting MEF2A as likely coronary artery disease-associated gene.}

Clinical phenotypes

Prolonged chest pain

ECG patterns consistent with acute myocardial infarction

Elevated cardiac enzymes

\section{Prior evidence available as ontology annotations}

MEF2A (WES candidate gene): cardiac conduction (G0:0061337), heart development (G0:0007507), ventricular cardiac myofibri assembly (G0:0055005), decreased heart rate (MP:0005333), dilated heart right ventricle (MP:0002754), premature death (MP:0002083)

\section{Selected interacting partner annotations}

CASP3: heart development (G0:0007507), platelet formation (GO:0030220), myocarditis pathway (PW:0001037), abnormal blood vessel morphology (MP:0001614), abnormal heart ventricle morphology (MP:0005294), dilated heart atrium (MP:0003140) CASP7: heart development (G0:0007507), abnormal heart ventricle morphology (MP:0005294), dilated heart atrium (MP:0003140) EP300: abnormal heart development (MP:0000267), abnormal heart valve morphology (MP:0000285), abnormal vasculogenesis (MP:0001622), arteritis (MP:0010138), atrial septal defect (MP:0010403), enlarged heart (MP:0000274) HDAC4: subaortic stenosis (HP:0001682), cardiac muscle hypertrophy in response to stress (G0:0014898) HDAC5: cardiac hypertrophy (MP:0001625), ventricular septal defect (MP:0010402)

HDAC9: cardiac hypertrophy (MP:0001625), heart left ventricle hypertrophy (MP:0002625), ventricular septal defect (MP:0010402) MAPK14: altered response to myocardial infarction (MP:0000343), cardiac fibrosis (MP:0003141), enlarged heart (MP:0000274),

increased response of heart to induced stress (MP:0004485)

MAPK7: abnormal heart morphology (MP:0000266)

MEF2D: adult heart development (G0:0007512), decreased cardiac muscle contractility (MP:0005140), premature death (MP:0002083)

MYOD 1: abnormal heart ventricle morphology (MP:0005294), cardiac fibrosis (MP:0003141), dilated cardiomyopathy (MP:0002795), increased heart weight (MP:0002833), myocardial necrosis (MP:0006085), premature death (MP:0002083)

SMAD4: aortic dilatation (HP:0001724), aortic valve stenosis (HP:0001650), EMG abnormality (HP:0003457), high-output congestive heart failure (HP:0001722), abnormal heart right ventricle morphology (MP:0003920)

THRA (thyroid hormone receptor, a): regulation of heart contraction (GO:0008016), abnormal heart rate (MP:0001629), prolonged QRS complex duration (MP:0010392), prolonged QT interval (MP:0003233) 
and complex diseases as well as data from animal disease models that can be downloaded or accessed via a web server (www.disgenet.org).

(iii) Does the gene have a function that coincides with the pathology of the disease of interest? Gene Ontology (GO) (42) annotations can be downloaded from: http://geneontology.org/page/downloadannotations, or a candidate gene's GO annotations can be searched using AMIGO: http://amigo.geneontology.org/amigo/ search/bioentity. To determine whether a set of functional variants contributes to functions relating to a phenotype of interest, genes can be assessed for enrichment of GO functions. The "gene set enricher" web service offered by the Comparative Toxicogenomics Database (43) is an intuitive tool for this purpose that also produces user-friendly outputs that can be imported directly into spreadsheet software for further filtering or manipulation (http://ctdbase.org/tools/analyzer. go). Additionally, Enricher (http://amp. pharm.mssm.edu/Enrichr) includes a wide

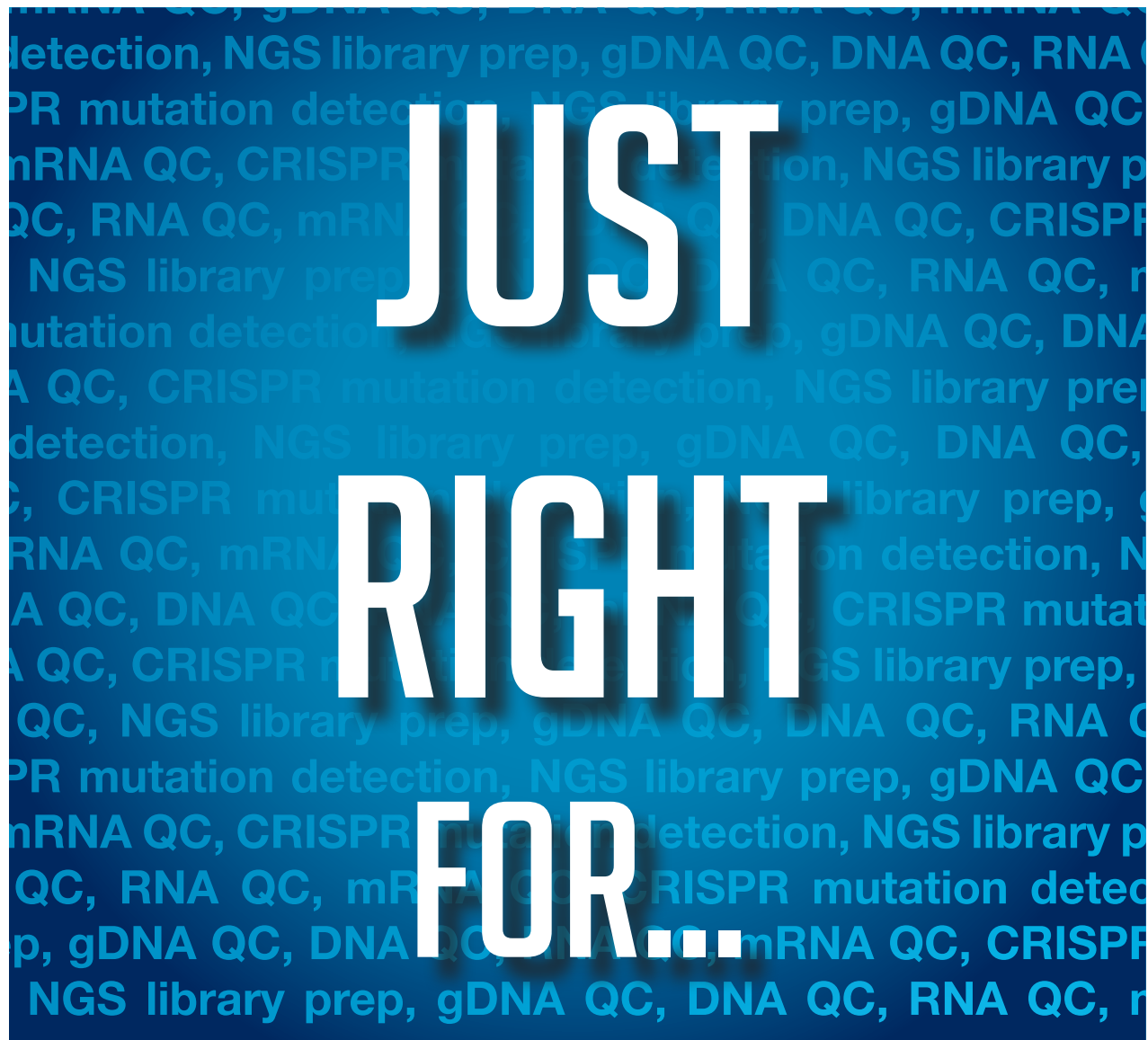

STREAMLINE GENOMIC WORKFLOWS WITH AUTOMATED NUCLEIC ACID QC

\begin{abstract}
The Fragment Analyzer ${ }^{\mathrm{TM}}$ is the premier instrument for automating QC steps in genomics workflow, from raw nucleic acids to final library validation.
\end{abstract}
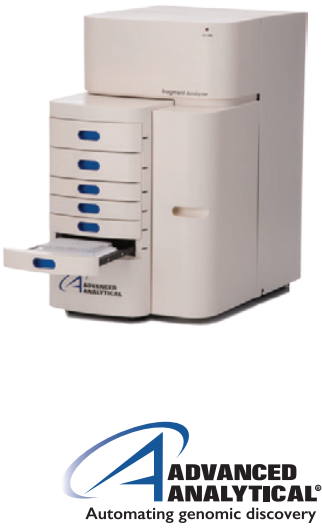

selection of libraries for collective functions of gene lists (44). Gene-set libraries include those derived from position weight matrices (PWMs) from TRANSFAC (45) and JASPAR (46), ENCODE transcription factor targets (47), histone modification data sets (48), microRNA targets (49), GeneSigDB (50) and MSigDB (51), VirusMINT (52), OMIM (53) and expanded OMIM (54), GO ontologies (42), WikiPathways (55), KEGG (56), Reactome (57), and kinase datasets (58).

(iv) Is the gene product in a pathway associated with the disease? Genes can be mapped online to KEGG pathways (www. genome.jp/kegg/) or REACTOME pathways (www.reactome.org/). Gene-to-pathway annotations can also be derived from the Pathway Ontology (59). As mentioned previously, it is possible to determine whether a set of variants collectively impact a pathway known to be associated with a disease or trait of interest by performing an enrichment analysis using the specific functionality and libraries in the Enricher web service (http:// amp.pharm.mssm.edu/Enrichr).

(v) Does a mutation or animal knockout of the gene cause the disease or a hallmark phenotype of the disease? The Mouse Genome Informatics database enables queries for human-mouse disease and Mammalian Phenotype Ontology (MPO) connections using gene symbols as an input (www.informatics.jax.org/humanDisease. shtml). The Human Phenotype Ontology (HPO) project (60) also provides gene-tophenotype mappings, which can be used in a similar manner (http://human-phenotypeontology.org). HPO plays a key role in the Monarch Initiative (61), which provides tools for genotype-phenotype analysis across broad areas of disease (https://monarchinitiative.org). Toolkits that have been built on top of these and other annotations primarily for the discovery of Mendelian genes include:

1. Exomiser (62), which has been shown to improve the prioritization of disease genes from WES variant calls through crossspecies phenotype comparisons (WwW. sanger.ac.uk/science/tools/exomiser).

2. PhenIX (Phenotypic Interpretation of eXomes), which ranks candidate genes in exomes based on predicted variant pathogenicity as well as the phenotypic similarity of diseases associated with the genes harboring these variants to the phenotypic profile of the individual(s) being investigated. 


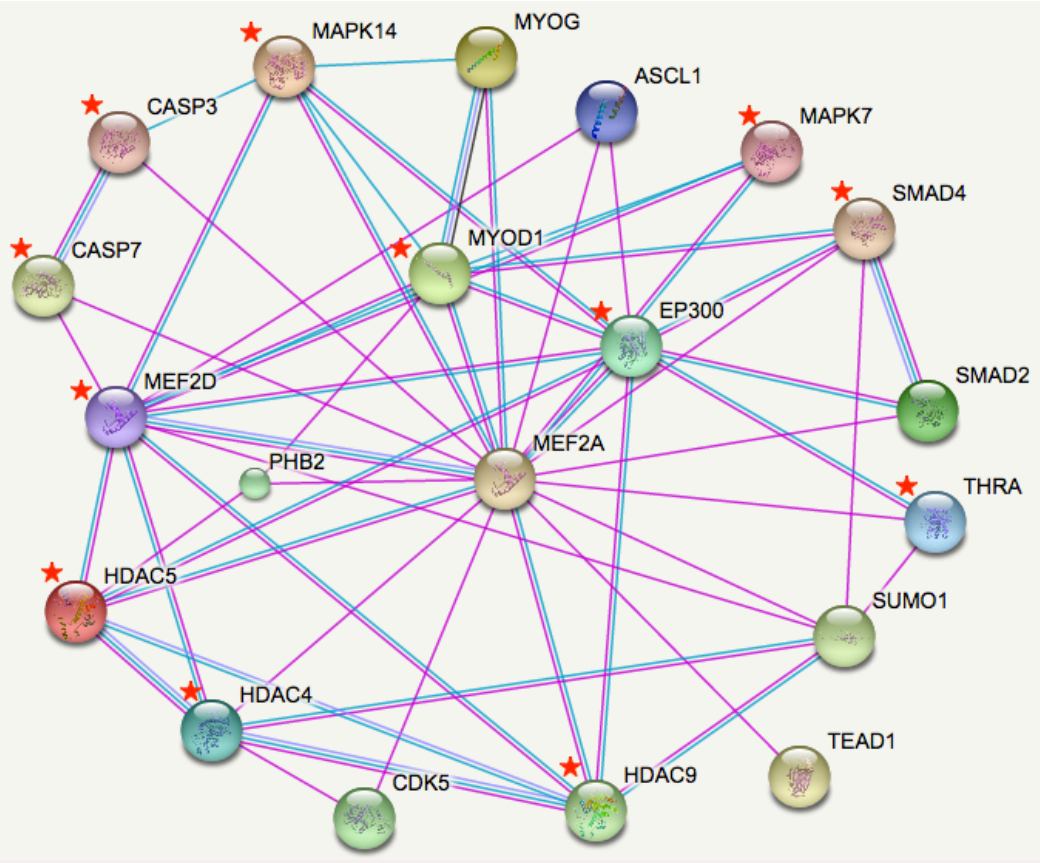

Figure 4. STRINGdb functional interaction network of the protein product of MEF2A. Red stars indicate proteins that have function, pathway, and phenotype annotations relevant to coronary artery disease.

Locally installable versions of this tool that offer functionality for pedigree filtering and prioritization based on other data sources are available (http:// compbio.charite.de/PhenIX).

3. OMIM Explorer (63), which uses semantic similarity and an adaptive approach for disease-gene discovery based on patient phenotypes to propose a computationally assisted differential diagnosis informed by a personal genome. This tool is particularly useful and intuitive as it allows the user to visually filter and update variant rankings by interacting with intermediate results (www.omimexplorer.com).

4. Exome Walker, which prioritizes candidate genes according to a variant score (predicted pathogenicity, rarity, assumed mode of inheritance) and their vicinity to other genes belonging to the same disease-gene family within the protein-protein interaction (PPI) network (http://compbio. charite.de/ExomeWalker).

5. Gene Distiller (64), which provides access to multiple knowledge and data sources, including HPO. Users can also interactively filter and prioritize candidate genes based on background biomedical knowledge and the clinical phenotype (www. genedistiller.org).
6. Phenolyzer (65), which uses prior knowledge to implicate genes involved in diseases based on disease or phenotype terms entered as free text (http://phenolyzer.usc.edu).

All of the above-listed tools use a VCF file as input, and while most are able to perform rankings based on raw VCF files, we recommend pre-filtering variants using VEP and study-specific criteria based on rules derived from Section A above. It is important to note that these tools require as complete a clinical phenotype as possible in order to make useful predictions. In addition to single-gene discovery approaches, phenotype-based statistical enrichment in gene sets can also be performed using evol.nhri.org.tw/phenome).

(vi) Is the gene expressed in the tissue or organ of interest? Gene Expression Omnibus profiles (www.ncbi.nlm.nih. gov/geoprofiles), the Expression Atlas (www.ebi.ac.uk/gxa), the BioGPS gene annotation portal (http://biogps.org), and the previously mentioned GTEx database (www.gtexportal.org/home/) are excellent resources for this purpose.

(vii) Is the gene intolerant to loss-offunction mutations? Function-impacting variants/mutations in genes that are never or only rarely mutated in healthy populations are more likely to be diseasethe MamPhEA (66) web server (http:// causing, and conversely, those in genes that are frequently mutated in healthy populations are unlikely to cause disease. At the same time, the latter are expected to be overrepresented in the genome of a given individual. It was recently shown that loss-of-function (LoF) intolerant genes include virtually all known severe haploinsufficient human disease genes (9), which suggests functional variants in this class of genes should be given higher priority in disease studies. Due to the large number of samples analyzed and the $>3000$ genes identified as being LoF-intolerant, of which $>70 \%$ have not yet been associated to a disease, we recommend using the "Functional Gene Constraint" scores from ExAC (http:// exac.broadinstitute.org/downloads) if implementing an automated filter or using the gene symbol or Ensembl ID as a seed for querying the ExAC Browser (http:// exac.broadinstitute.org/). A gene with a LoF tolerance score [probability of being loss-of-function intolerant ( $\mathrm{pLI})] \geq 0.9$ is considered extremely LoF intolerant. The Human Gene Damage Index server (http://lab.rockefeller.edu/casanova/GDI) is another option for identifying LoF-intolerant genes (67). It is important to note that LoF-intolerance should not be the only (or even the primary) filtering criterion.

(viii) Does the gene product physically interact with a protein encoded by a known disease gene? Much like genes encoding proteins that occur in the same pathway as a known diseasegene product may also cause the disease if mutated, so too may proteins that physically interact with known disease-gene products. The STRING database and associated search tools (68) are powerful resources for identifying interacting partners of a candidate gene's product or to identify interactions between the products of a set of genes that bear functional variants (http://string-db. org). The ToppGene (https://toppgene. cchmc.org) (69) and GeneMania (http:// genemania.org) (70) web portals are other resources that perform candidate gene prioritization based on the interactome. The relevance of this prioritization strategy is illustrated in an example below, where we show how functional loss of genes encoding several of the direct interaction partners of the product of a disease-gene candidate are themselves known to cause key phenotypes of the disease. 


\section{Extant-knowledge-based candidate prioritization}

We examined the existing biological and biomedical evidence to clarify the ambiguity surrounding the identification of MEF2A as a disease gene for premature coronary artery disease, as suggested in a recent pedigree-based WES study (55). While previous reports suggested the gene was associated with the disease, subsequent multiple-family studies, as well as analysis of another 1700 sporadic cases of the disease, could not reproduce the linkage. It is important to note that this example is presented to illustrate the value of using extant knowledge in the candidate gene vetting process, and does not constitute support of the gene's candidacy.

To evaluate the MEF2A's potential involvement in the disease, we first identified its interacting partners in STRINGdb. Next, we annotated these partners with gene (GO), human phenotype (HPO), mammalian phenotype (MPO), and pathway (PW) annotations using the above-mentioned resources. These were then crosschecked against the described clinical phenotypes of "prolonged chest pain," "electrocardiogram patterns consistent with acute myocardial infarction," and "elevation of cardiac enzymes," to form a hypothesis about the gene's potential role in the disease. Table 1 summarizes our findings supporting the conclusion that mutations in the MEF2A gene probably explain the clinical phenotype, particularly since the variant perfectly segregates with the affected individuals in the family, which as we recommend, should carry greater weight than its likely functional impact. The value of assessing interacting partners is also illustrated here, as 12 of 19 have annotations relevant to the disease (Figure 4), giving clues about the likely cardiac role of MEF2A even if it itself had no relevant annotations.

\section{Conclusion}

The guidelines presented here put variant prioritization well within the reach of biologists and clinicians who may not have formal bioinformatics training. Extant knowledge about the molecular and cellular mechanisms involved in a disease or phenotype of interest can be extremely useful in efforts to prioritize likely candidates from a list of genes possessing functional variants. Rarity/ novelty along with predicted deleterious effects and expected segregation with affected/cases and unaffected/control individuals in the study are the primary criteria for producing a candidate list. Therefore, variants should not be discarded as being irrelevant if the knowledge filter does not return disease, phenotypic, or functional links. It is also important to note that not all of the abovementioned questions will yield useful information. However, in many cases, the insights gained from thoroughly interrogating one knowledge domain provide enough evidence to implicate a variant. Additionally, careful consideration should be given to the drawbacks associated with each tool developed to allow the assessment of variants in the context of a disease phenotype. All have limitations; thus, we recommend that a combination of tools should be used where possible.

While the filters described here act as a funnel, it is important to note that (i) not all steps may be relevant, (ii) each filter should be customized to the study requirements, and (iii) the funnel will not always comprise all of the possible filters. Although a variant that fulfills multiple criteria is most likely to have a genotypeto-phenotype association, this should not be an absolute criterion for determining its candidacy.
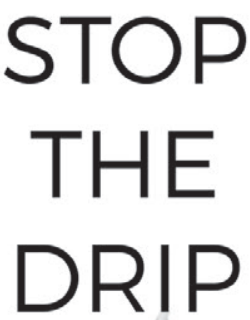

Available through distributors

Available through distributors
For more information go to ww
The Better Way to Pipette

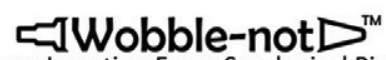

Low Insertion Force Serological Pipet

No Drip Design

2 -tiers $/ 2$ steps $=2 \mathrm{X}$ contact in your controller $=$ better seal

For Hassle-Free Serological Pipetting

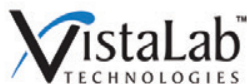

Think DHNOLOG IES

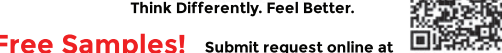

www.vistalab.com/wobble-not/\#get-free-sample-pack
w

\section{Acknowledgments}

The work done for this manuscript was partially supported by a South African Medical Research Council Strategic Health Innovation Partnerships (SHIP) grant and by National Institutes of Health $(\mathrm{NIH})$ grant $\mathrm{U} 41 \mathrm{HG} 006941$. This paper is subject to the NIH Public Access Policy.

\section{Competing interests}

The authors declare no competing interests.

\section{References}

1. Choi, M., U.I. Scholl, W. Ji, T. Liu, I.R. Tikhonova P. Zumbo, A. Nayir, A. Bakkaloglu, et al. 2009. Genetic diagnosis by whole exome capture and massively parallel DNA sequencing. Proc. Natl. Acad. Sci. USA 106:19096-19101.

2. Bilgüvar, K., A.K. Oztürk, A. Louvi, K.Y. Kwan, M. Choi, B. Tatli, D. Yalnizoglu, B. Tüysüz, et al. 2010. Whole-exome sequencing identifies recessive WDR62 mutations in severe brain malformations. Nature 467:207-210.

3. Johnston, J.J., J.K. Teer, P.F. Cherukuri, N.F. Hansen, S.K. Loftus, K. Chong, J.C. Mullikin, and L.G. Biesecker. 2010. Massively parallel sequencing of exons on the X chromosome identifies RBM10 as the gene that causes a syndromic form of cleft palate. Am. J. Hum. Genet. 86:743-748.

4. Ng, S.B., A.W. Bigham, K.J. Buckingham, M.C. Hannibal, M.J. McMillin, H.I. Gildersleeve, A.E. Beck, H.K. Tabor, et al. 2010. Exome sequencing identifies MLL2 mutations as a cause of Kabuki syndrome. Nat. Genet. 42:790-793.

5. Erlich, Y., S. Edvardson, E. Hodges, S. Zenvirt, P. Thekkat, A. Shaag, T. Dor, G.J. Hannon, and O. Elpeleg. 2011. Exome sequencing and diseasenetwork analysis of a single family implicate a mutation in KIF1A in hereditary spastic paraparesis. Genome Res. 21:658-664.

6. Miosge, L.A., M.A. Field, Y. Sontani, V. Cho, S. Johnson, A. Palkova, B. Balakishnan, R. Liang, et al. 2015. Comparison of predicted and actual consequences of missense mutations. Proc. Natl. Acad. Sci. USA 112:E5189-E5198.

7. MacArthur, D.G., S. Balasubramanian, A. Frankish, N. Huang, J. Morris, K. Walter, L. Jostins, L. Habegger, et al. 2012. A systematic survey of loss-of-function variants in human proteincoding genes. Science 335:823-828.

8. Richards, S., N. Aziz, S. Bale, D. Bick, S. Das, J. Gastier-Foster, W.W. Grody, M. Hegde, et al. 2015. Standards and guidelines for the interpretation of sequence variants: a joint consensus recommendation of the American College of Medical Genetics and Genomics and the Association for Molecular Pathology. Genet. Med. 17:405-424.

9. Lek, M., K.J. Karczewski, E.V. Minikel, K.E. Samocha, E. Banks, T. Fennell, A.H. O'DonnellLuria, J.S. Ware, et al. 2016. Analysis of proteincoding genetic variation in 60,706 humans. Nature 536:285-291.

10. McCarthy, D.J., P. Humburg, A. Kanapin, M.A. Rivas, K. Gaulton, J.-B. Cazier, and P. Donnelly. 2014. Choice of transcripts and software has a large effect on variant annotation. Genome Med. 6:26.

11. O'Leary, N.A., M.W. Wright, J.R. Brister, S. Ciufo, D. Haddad, R. McVeigh, B. Rajput, B. Robbertse, 
et al. 2016. Reference sequence (RefSeq) database at NCBI: current status, taxonomic expansion, and functional annotation. Nucleic Acids Res. 44:D733D745.

12. Yates, A., W. Akanni, M.R. Amode, D. Barrell, K. Billis, D. Carvalho-Silva, C. Cummins, P. Clapham, et al. 2016. Ensembl 2016. Nucleic Acids Res. 44:D710-D716.

13. Cingolani, P., A. Platts, L.L. Wang, M. Coon, T. Nguyen, L. Wang, S.J. Land, X. Lu, and D.M. Ruden. 2012. A program for annotating and predicting the effects of single nucleotide polymorphisms, SnpEff. Fly (Austin) 6:80-92.

14. Wang, K., M. Li, and H. Hakonarson. 2010. ANNOVAR: functional annotation of genetic variants from high-throughput sequencing data. Nucleic Acids Res. 38:e164

15. McLaren, W., L. Gil, S.E. Hunt, H.S. Riat, G.R.S. Ritchie, A. Thormann, P. Flicek, and F. Cunningham. 2016. The Ensembl Variant Effect Predictor. Genome Biol. 17:122.

16. Liu, X., X. Jian, and E. Boerwinkle. 2011. dbNSFP: a lightweight database of human nonsynonymous SNPs and their functional predictions. Hum. Mutat. 32:894-899.

17. McKenna, A., M. Hanna, E. Banks, A. Sivachenko, K. Cibulskis, A. Kernytsky, K. Garimella, D. Altshuler, et al. 2010. The Genome Analysis Toolkit: a MapReduce framework for analyzing next-generation DNA sequencing data. Genome Res. 20:1297-1303.

18. MacArthur, D.G., T.A. Manolio, D.P. Dimmock, H.L. Rehm, J. Shendure, G.R. Abecasis, D.R. Adams, R.B. Altman, et al. 2014. Guidelines for investigating causality of sequence variants in human disease. Nature 508:469-476.

19. Aschard, H., W. Qiu, B. Pasaniuc, N. Zaitlen, M.H. Cho, and V. Carey. 2011. Combining effects from rare and common genetic variants in an exome-wide association study of sequence data. BMC Proc. 5(Supp/ 9):S44.

20. Hu, H., C.D. Huff, B. Moore, S. Flygare, M.G. Reese, and M. Yandell. 2013. VAAST 2.0: Improved variant classification and disease-gene identification using a conservation-controlled amino acid substitution matrix. Genet. Epidemiol. 37:622-634.

21. Paila, U., B.A. Chapman, R. Kirchner, A.R. Quinlan, A. McKenna, M. Hanna, E. Banks, A. Sivachenko, et al. 2013. GEMINI: Integrative Exploration of Genetic Variation and Genome Annotations. PLOS Comput. Biol. 9:e1003153.

22. Li, M.-X., H.-S. Gui, J.S.H. Kwan, S.-Y. Bao, and P.C. Sham. 2012. A comprehensive framework for prioritizing variants in exome sequencing studies of Mendelian diseases. Nucleic Acids Res. 40:e53.

23. Wei, L., L.T. Liu, J.R. Conroy, Q. Hu, J.M. Conroy, C.D. Morrison, C.S. Johnson, J. Wang, et al. 2015. MAC: identifying and correcting annotation for multi-nucleotide variations. BMC Genomics 16:569.

24. Nelson, M.R., D. Wegmann, M.G. Ehm, D. Kessner, P. St Jean, C. Verzilli, J. Shen, Z. Tang, et al. 2012. An abundance of rare functional variants in 202 drug target genes sequenced in 14,002 people. Science 337:100-104.

25. Cooper, D.N., M. Krawczak, C. Polychronakos, C. Tyler-Smith, and H. Kehrer-Sawatzki. 2013. Where genotype is not predictive of phenotype: towards an understanding of the molecular basis of reduced penetrance in human inherited disease. Hum. Genet. 132:1077-1130.

26. Lazarin, G.A., I.S. Haque, S. Nazareth, K. Iori, A.S. Patterson, J.L. Jacobson, J.R. Marshall, W.K. Seltzer, et al. 2013. An empirical estimate of carrier frequencies for $400+$ causal Mendelian variants: results from an ethnically diverse clinical sample of 23,453 individuals. Genet. Med. 15:178186.

27. Jordan, D.M., S.G. Frangakis, C. Golzio, C.A. Cassa, J. Kurtzberg, E.E. Davis, S.R. Sunyaev, and N. Katsanis. 2015. Identification of cis-suppression of human disease mutations by comparative genomics. Nature 524:225-229.

28. Ng, P.C. and S. Henikoff. 2003. SIFT: Predicting amino acid changes that affect protein function. Nucleic Acids Res. 31:3812-3814.

29. Adzhubei, I., D.M. Jordan, and S.R. Sunyaev. 2013. Predicting functional effect of human missense mutations using PolyPhen-2. Curr Protoc Hum Genet. Chapter 7:Unit7.20.

30. Flanagan, S.E., A.-M. Patch, and S. Ellard. 2010. Using SIFT and PolyPhen to predict loss-of-function and gain-of-function mutations. Genet. Test. Mol. Biomarkers 14:533-537.

31. Dong, C., P. Wei, X. Jian, R. Gibbs, E. Boerwinkle, K. Wang, and X. Liu. 2015. Comparison and integration of deleteriousness prediction methods for nonsynonymous SNVs in whole exome sequencing studies. Hum. Mol. Genet. 24:2125-2137.

32. Hu, J. and P.C. Ng. 2013. SIFT Indel: Predictions for the Functional Effects of Amino Acid Insertions/ Deletions in Proteins. PLoS One 8:e77940.

33. Mitchell, A., H.-Y. Chang, L. Daugherty, M. Fraser, S. Hunter, R. Lopez, C. McAnulla, C. McMenamin, et al. 2015. The InterPro protein families database: the classification resource after 15 years. Nucleic Acids Res. 43:D213-D221.

34. Lee, S.Y., M.J. Hong, H.-S. Jeon, Y.Y. Choi, J.E. Choi, H.-G. Kang, D.K. Jung, C. Jin, et al. 2015. Functional intronic ERCC1 polymorphism from regulomeDB can predict survival in lung cancer after surgery. Oncotarget 6:24522-24532.

35. Boyle, A.P., E.L. Hong, M. Hariharan, Y. Cheng, M.A. Schaub, M. Kasowski, K.J. Karczewski, J. Park, et al. 2012. Annotation of functional variation in personal genomes using RegulomeDB. Genome Res. 22:1790-1797.

36. GTEx Consortium. 2015. Human genomics. The Genotype-Tissue Expression (GTEx) pilot analysis: multitissue gene regulation in humans. Science 348:648-660.

37. Shihab, H.A., M.F. Rogers, J. Gough, M. Mort, D.N. Cooper, I.N.M. Day, T.R. Gaunt, and C. Campbell. 2015. An integrative approach to predicting the functional effects of non-coding and coding sequence variation. Bioinformatics 31:15361543.

38. Kircher, M., D.M. Witten, P. Jain, B.J. O’Roak G.M. Cooper, and J. Shendure. 2014. A general framework for estimating the relative pathogenicity of human genetic variants. Nat. Genet. 46:310-315.

39. Zhou, J. and O.G. Troyanskaya. 2015. Predicting effects of non-coding variants with deep learningbased sequence model. Nat. Methods 12:931-934.

40. Landrum, M.J., J.M. Lee, G.R. Riley, W. Jang, W.S. Rubinstein, D.M. Church, and D.R. Maglott.

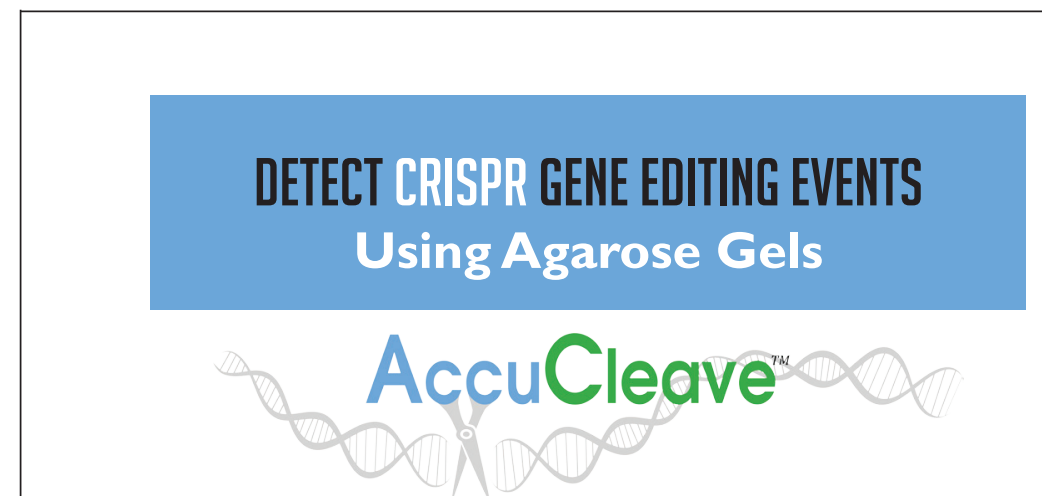

\section{Streamline gene editing workflows with a simple and accurate enzymatic assay.}

\section{AccuCleave ${ }^{\mathrm{TM}}$ T7 Kit}

- T7 based enzyme kit for mutation frequency detection of CRISPR events.

\section{AccuCleave ${ }^{\mathrm{TM}} \mathrm{C} 2$ Control DNA Kit \\ - Contains 2 Control DNA fragments - intact and -2 deletion fragment.}

\section{AccuCleave ${ }^{\mathrm{TM}}$ CI5 Control DNA Kit \\ - Contains 15 Control DNA fragments - intact, $\pm \mathrm{I}, \pm 2, \pm 10 \mathrm{InDels}$, and 8 point mutations.}

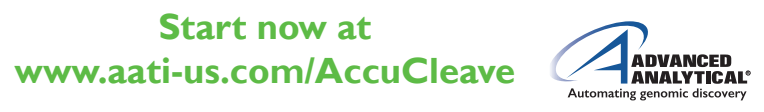




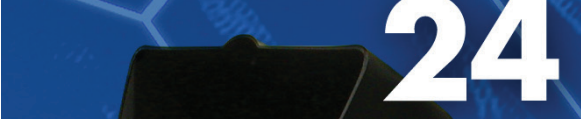

8

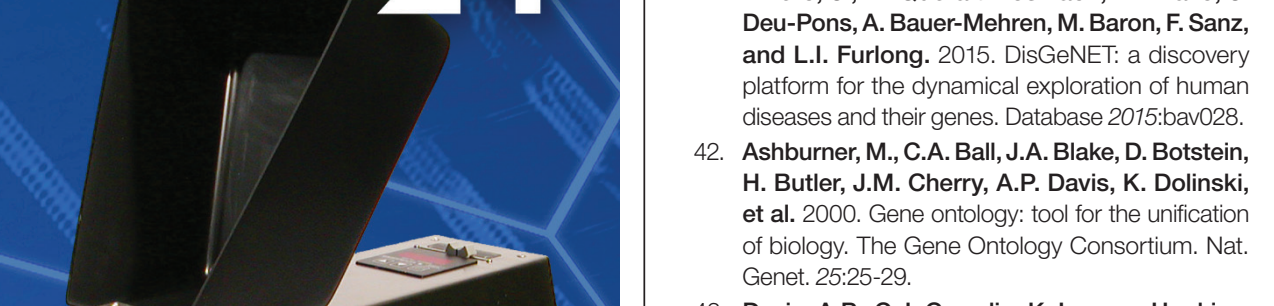

.

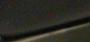
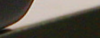

The Mini-Beadbeater-24 disrupts microorganisms and tissue preparations with better than 95 percent efficiency. Nucleic acids and proteins are isolated with the highest possible yields. Cells are disrupted quickly and safely in the sealed $2 \mathrm{ml}$ microvials containing small beads. No cleaning between samples is required The apparatus has a small footprint and is essentially maintenance free.

- Uses proven, more efficient near horizontal vial shaking motion.

- $24-2.0 \mathrm{ml}$ microvials capacity with removable vial-holding cassette.

- Variable shaking speed:2000 - 3450 strokes/min.

- Digital 5 minute timer with auto reset.

- No imposed motor cool-downtime between each sample run.

- Best price.

BSE BIOSPEC PRODUCTS

P.O. Box 788 Bartlesville, OK 74005

800-617-3363 www.biospec.com
2014. ClinVar: public archive of relationships among sequence variation and human phenotype. Nucleic Acids Res. 42:D980-D985.

41. Piñero, J., N. Queralt-Rosinach, À. Bravo, J. Deu-Pons, A. Bauer-Mehren, M. Baron, F. Sanz, and L.I. Furlong. 2015. DisGeNET: a discovery platform for the dynamical exploration of human diseases and their genes. Database 2015:bav028. et al. 2000 of biology. The Gene Ontology Consortium. Nat.

. Davis, A.P., C.J. Grondin, K. Lennon-Hopkins, C. Saraceni-Richards, D. Sciaky, B.L. King, T.C. Wiegers, and C.J. Mattingly. 2015. The Comparative Toxicogenomics Database's 10th year anniversary: update 2015. Nucleic Acids Res. 43:D914-D920.

44. Chen, E.Y., C.M. Tan, Y. Kou, Q. Duan, Z. Wang, G.V. Meirelles, N.R. Clark, and A. Ma'ayan. 2013. Enrichr: interactive and collaborative HTML5 gene list enrichment analysis tool. BMC Bioinformatics 14:128.

45. Matys, V., O.V. Kel-Margoulis, E. Fricke, I. Liebich, S. Land, A. Barre-Dirrie, I. Reuter, D. Chekmenev, et al. 2006. TRANSFAC and its module TRANSCompel: transcriptional gene regulation in eukaryotes. Nucleic Acids Res. 34:D108-D110.

46. Portales-Casamar, E., S. Thongjuea, A.T. Kwon, D. Arenillas, X. Zhao, E. Valen, D. Yusuf, B. Lenhard, et al. 2010. JASPAR 2010: the greatly expanded open-access database of transcription factor binding profiles. Nucleic Acids Res. 38:D105D110.

47. Rosenbloom, K.R., T.R. Dreszer, J.C. Long, V.S. Malladi, C.A. Sloan, B.J. Raney, M.S. Cline, D. Karolchik, et al. 2012. ENCODE whole-genome data in the UCSC Genome Browser: update 2012. Nucleic Acids Res. 40:D912-D917.

48. Bernstein, B.E., J.A. Stamatoyannopoulos, J.F. Costello, B. Ren, A. Milosavljevic, A. Meissner, M. Kellis, M.A. Marra, et al. 2010. The NIH Roadmap Epigenomics Mapping Consortium. Nat. Biotechnol. 28:1045-1048

49. Lewis, B.P., I. Shih, M.W. Jones-Rhoades, D.P. Bartel, and C.B. Burge. 2003. Prediction of mammalian microRNA targets. Cell 115:787-798.

50. 50. Culhane, A.C., T. Schwarzl, R. Sultana, K.C. Picard, S.C. Picard, T.H. Lu, K.R. Franklin, S.J. French, et al. 2010. GeneSigDB--a curated database of gene expression signatures. Nucleic Acids Res. 38:D716-D725.

51. Liberzon, A., A. Subramanian, R. Pinchback, H. Thorvaldsdóttir, P. Tamayo, and J.P. Mesirov. 2011. Molecular signatures database (MSigDB) 3.0. Bioinformatics 27:1739-1740.

52. Chatr-aryamontri, A., A. Ceol, D. Peluso, A. Nardozza, S. Panni, F. Sacco, M. Tinti, A. Smolyar, et al. 2009. VirusMINT: a viral protein interaction database. Nucleic Acids Res. 37:D669-D673.

53. Hamosh, A., A.F. Scott, J. Amberger, D. Valle, and V.A. McKusick. 2000. Online Mendelian Inheritance in Man (OMIM). Hum. Mutat. 15:57-61.

54. Berger, S.I., J.M. Posner, and A. Ma'ayan. 2007. Genes2Networks: connecting lists of gene symbols using mammalian protein interactions databases. BMC Bioinformatics 8:372.

55. Pico, A.R., T. Kelder, M.P. van lersel, K. Hanspers, B.R. Conklin, and C. Evelo. 2008. WikiPathways: pathway editing for the people. PLoS Biol. 6:e184.

56. Kanehisa, M., Y. Sato, M. Kawashima, M. Furumichi, and M. Tanabe. 2016. KEGG as a reference resource for gene and protein annotation. Nucleic Acids Res. 44:D457-D462.

57. Fabregat, A., K. Sidiropoulos, P. Garapati, M. Gillespie, K. Hausmann, R. Haw, B. Jassal, S. Jupe, et al. 2016. The Reactome pathway Knowledgebase. Nucleic Acids Res. 44:D481-D487.

58. Lachmann, A. and A. Ma'ayan. 2009. KEA: kinase enrichment analysis. Bioinformatics 25:684-686.

59. Petri, V., P. Jayaraman, M. Tutaj, G.T. Hayman, J.R. Smith, J. De Pons, S.J. Laulederkind, T.F. Lowry, et al. 2014. The pathway ontology - updates and applications. J. Biomed. Semantics 5:7.

60. Groza, T., S. Köhler, D. Moldenhauer, N. Vasilevsky, G. Baynam, T. Zemojtel, L.M. Schriml, W.A. Kibbe, et al. 2015. The Human Phenotype Ontology: Semantic Unification of Common and Rare Disease. Am. J. Hum. Genet. 97:111-124.

61. McMurry, J.A., S. Köhler, N.L. Washington, J.P. Balhoff, C. Borromeo, M. Brush, S. Carbon, T. Conlin, et al. 2016. Navigating the Phenotype Frontier: The Monarch Initiative. Genetics 203:14911495.

62. Bone, W.P., N.L. Washington, O.J. Buske, D.R. Adams, J. Davis, D. Draper, E.D. Flynn, M. Girdea, et al. 2016. Computational evaluation of exome sequence data using human and model organism phenotypes improves diagnostic efficiency. Genet. Med. 18:608-617.

63. James, R.A., I.M. Campbell, E.S. Chen, P.M. Boone, M.A. Rao, M.N. Bainbridge, J.R. Lupski, Y. Yang, et al. 2016. A visual and curatorial approach to clinical variant prioritization and disease gene discovery in genome-wide diagnostics. Genome Med. 8:13.

64. Seelow, D., J.M. Schwarz, and M. Schuelke. 2008. GeneDistiller--distilling candidate genes from linkage intervals. PLoS One 3:e3874.

65. Yang, H., P.N. Robinson, and K. Wang. 2015. Phenolyzer: phenotype-based prioritization of candidate genes for human diseases. Nat. Methods 12:841-843.

66. Weng, M.-P. and B.-Y. Liao. 2010. MamPhEA: a web tool for mammalian phenotype enrichment analysis. Bioinformatics 26:2212-2213.

67. Itan, Y., L. Shang, B. Boisson, E. Patin, A. Bolze, M. Moncada-Vélez, E. Scott, M.J. Ciancanelli, et al. 2015. The human gene damage index as a genelevel approach to prioritizing exome variants. Proc. Natl. Acad. Sci. USA 112:13615-13620.

68. Szklarczyk, D., A. Franceschini, S. Wyder, K. Forslund, D. Heller, J. Huerta-Cepas, M. Simonovic, A. Roth, et al. 2015. STRING v10: protein-protein interaction networks, integrated over the tree of life. Nucleic Acids Res. 43:D447-D452.

69. Chen, J., E.E. Bardes, B.J. Aronow, and A.G. Jegga. 2009. ToppGene Suite for gene list enrichment analysis and candidate gene prioritization. Nucleic Acids Res. 37:W305-W311.

70. Zuberi, K., M. Franz, H. Rodriguez, J. Montojo, C.T. Lopes, G.D. Bader, and Q. Morris. 2013. GeneMANIA prediction server 2013 update. Nucleic Acids Res. 41:W115-W122.

Received 22 November 2015; accepted 11 October 2016.

Address correspondence to Junaid Gamieldien, South African National Bioinformatics Institute, University of the Western Cape, Robert Sobukwe Road, Bellville 7530, South Africa. E-mail: junaid@sanbi.ac.za

To purchase reprints of this article, contact: biotechniques@fosterprinting.com 